PROCEEDINGS OF THE

AMERICAN MATHEMATICAL SOCIETY

Volume 134, Number 12, December 2006, Pages 3645-3648

S 0002-9939(06)08422-X

Article electronically published on June 13, 2006

\title{
A MYERS-TYPE THEOREM AND COMPACT RICCI SOLITONS
}

\author{
ANDRZEJ DERDZINSKI
}

(Communicated by Jon G. Wolfson)

\begin{abstract}
Let the Ricci curvature of a compact Riemannian manifold be greater, at every point, than the Lie derivative of the metric with respect to some fixed smooth vector field. It is shown that the fundamental group then has only finitely many conjugacy classes. This applies, in particular, to all compact shrinking Ricci solitons.
\end{abstract}

\section{INTRODUCTION}

Myers's classical theorem [1, p. 165] implies that any compact Riemannian manifold with positive Ricci curvature has a finite fundamental group. Its standard proof uses a diameter estimate. In this note we observe that an easy upper bound (4) on the length spectrum leads to a similar, though seemingly weaker, assertion, valid in a more general case.

Theorem 1. If $(M, g)$ is a compact Riemannian manifold such that

$$
\mathcal{L}_{w} g+\text { Ric }>0
$$

for some $C^{\infty}$ vector field $w$, then $\pi_{1} M$ has only finitely many conjugacy classes.

Here $\mathcal{L}_{w}$ and Ric are the Lie derivative and the Ricci tensor, while positivity in (11) means positive-definiteness at every point. As $H_{1}(M, \mathbf{Z})=\pi_{1} M /\left[\pi_{1} M, \pi_{1} M\right]$, the following corollary is immediate:

Corollary 2. For any compact Riemannian manifold $(M, g)$ satisfying the assumptions of Theorem 1, the homology group $H_{1}(M, \mathbf{Z})$ is finite and $b_{1}(M)=0$.

It is not known if the conclusion of Theorem 1 is actually weaker than finiteness of $\pi_{1} M$. Whether a finitely presented group with only finitely many conjugacy classes must itself be finite is an open question, raised by Makowsky [1] in 1974. Another part of Myers's theorem, which states that a complete Riemannian manifold satisfying the condition Ric $\geq c g>0$ for a constant $c$ is necessarily compact, fails when Ric is replaced by $\mathcal{L}_{w} g+$ Ric. Namely, some noncompact complete Riemannian manifolds $(M, g)$ admit $C^{\infty}$ vector fields $w$ with

$$
\mathcal{L}_{w} g+\text { Ric }=c g \text { for a constant } c
$$

such that $c>0$, and so $\mathcal{L}_{w} g+$ Ric $\geq c g>0$. The simplest example is provided by a Euclidean space $(M, g)$ with the radial (identity) vector field $w$. Feldman,

Received by the editors December 8, 2004 and, in revised form, July 11, 2005.

2000 Mathematics Subject Classification. Primary 53C25; Secondary 53C20.

(C)2006 American Mathematical Society Reverts to public domain 28 years from publication 
Ilmanen and Knopf [4, Theorem 1.5] found further such examples, in which $(M, g)$ are Kähler manifolds and the vector fields $w$ are real holomorphic.

A Riemannian manifold $(M, g)$ admitting a $C^{\infty}$ vector field $w$ with (2) is called a Ricci soliton [7]; one then also refers to $g$ as a quasi-Einstein metric [6]. A Ricci soliton $(M, g)$ is said to be shrinking if $c>0$ in (2). See also $\$ 3$

Known examples of compact Ricci solitons consist of compact Einstein manifolds, two classes of non-Einstein Kähler Ricci solitons on compact complex manifolds (one constructed by Koiso [10] and, independently, Cao 2], the other by Wang and Zhu [13]), and, finally, suitable Riemannian products of the above. A self-contained exposition of results on compact Ricci solitons is given in [3].

The assumptions (and hence conclusions) of Theorem 1 and Corollary 2 clearly hold when $(M, g)$ is a compact shrinking Ricci soliton, or a small perturbation thereof. For compact shrinking Ricci solitons $(M, g)$, some special cases of this are known 1 finiteness of $H_{1}(M, \mathbf{Z})$ is a trivial consequence of [8, Theorem 1] (see 93 ); $M$ is simply connected if $g$ is also a Kähler metric (\$3); finally, Friedan [6] p. 396] states that $b_{1}(M)=0$ under the additional assumption of a scalar curvature bound Scal $\geq(\operatorname{dim} M-2) c$, for $c$ as in (2).

As shown by Ivey [9, Proposition 1] (cf. also [6. p. 396], and [3]), every non-Einstein compact Ricci soliton is a shrinking soliton. This leads to a further conclusion.

Corollary 3. The Euler characteristic of every non-flat compact four-dimensional Ricci soliton is positive.

In other words, Berger's inequality $\chi(M)>0$, for the Euler characteristic of a non-flat compact four-dimensional Einstein manifold [1, Theorem 6.32], remains true for Ricci solitons $(M, g)$. In fact, $\chi(M)=2+b_{2}(M)$ in the (orientable) nonEinstein case, since the soliton is shrinking and so, as stated above, $b_{1}(M)=0$.

The author wishes to thank Tadeusz Januszkiewicz, Gideon Maschler, Ronald Solomon, Fangyang Zheng and the referee for helpful comments.

\section{A simple estimate}

Given $a, b \in \mathbf{R}$ with $a<b$, let a constant-speed geodesic $[a, b] \ni t \mapsto x(t)$ in an orientable Riemannian manifold $(M, g)$ be smoothly closed, so that $x(b)=x(a)$ and $\dot{x}(b)=\dot{x}(a)$, and have the minimum length compared to all nearby smoothly closed $C^{\infty}$ curves $[a, b] \rightarrow M$. Then

$$
(b-a) \int_{a}^{b} \operatorname{Ric}(\dot{x}, \dot{x}) d t \leq 2[\operatorname{dist}(I, \Psi)]^{2} \leq k \pi^{2},
$$

where $k$ is the largest even integer with $k+1 \leq \operatorname{dim} M$, while $\Psi \in \operatorname{SO}(m)$, for $m=\operatorname{dim} M-1$, is a holonomy matrix of our smoothly closed geodesic, characterized by the matrix-product relation $\left[e_{1}(a) \ldots e_{m}(a)\right]=\left[e_{1}(b) \ldots e_{m}(b)\right] \Psi$ for a system $[a, b] \ni t \mapsto e_{j}(t) \in T_{x(t)} M$ of $m$ orthonormal vector fields parallel along the geodesic and normal to it. In addition, $I \in \mathrm{SO}(m)$ is the identity matrix, and dist stands for the geodesic distance function in $\mathrm{SO}(m)$ corresponding to its

\footnotetext{
${ }^{1}$ The present paper was released as a preprint (arXiv:math.DG/0403052) in March 2004. Shortly afterwards Fernández-López and García-Río [5] showed that condition (1) on a compact manifold implies finiteness of the fundamental group, and, in particular, the fundamental group of a compact shrinking Ricci soliton must be finite. I wish to thank Eduardo García-Río for sharing this information with me.
} 
submanifold metric induced by the inner product $Q$ in the ambient vector space $\mathfrak{g l}(m, \mathbf{R})$ of all $m \times m$ real matrices, given by $2 Q(\Psi, \Phi)=\operatorname{tr} \Psi \Phi^{*}$.

We now verify (3). In any Riemannian manifold $(M, g)$, if $t \mapsto u(t)$ is a $C^{\infty}$ unit vector field normal to a fixed geodesic $[a, b] \ni t \mapsto x(t)$ having the properties listed in the lines preceding (3), and $u(b)=u(a)$, then $(R(\dot{x}, u) \dot{x}, u) \leq(\dot{u}, \dot{u})$, where $(u, v)=\int_{a}^{b}\langle u, v\rangle d t$ stands for the $L^{2}$ inner product of vector fields $u, v$ tangent to $M$ along the geodesic, $R$ is the curvature tensor, and $\dot{u}=\nabla_{\dot{x}} u$. This is a wellknown consequence of the length-minimizing property of the geodesic; cf. 8 , formula (3.1)]. Let us now select the $m$ fields $e_{j}$ as in the lines following (3). For any fixed $C^{\infty}$ curve $[a, b] \ni t \mapsto \Phi(t) \in \mathrm{SO}(m)$ joining $I$ to the holonomy matrix $\Psi$, we may apply the inequality $(R(\dot{x}, u) \dot{x}, u) \leq(\dot{u}, \dot{u})$ to each of the $m$ fields $u=u_{j}$ given by the matrix-product formula $\left[u_{1}(t) \ldots u_{m}(t)\right]=\left[e_{1}(t) \ldots e_{m}(t)\right] \Phi(t)$. Summing the resulting inequalities over $j=1, \ldots, m$, we get $\int_{a}^{b} \operatorname{Ric}(\dot{x}, \dot{x}) d t \leq 2 \int_{a}^{b}|\dot{\Phi}|^{2} d t$, with || corresponding to the inner product $Q$. Since the curve $t \mapsto \Phi(t)$ in $\mathrm{SO}(m)$ is arbitrary, we may choose it to be a constant-speed minimizing geodesic joining $I$ to $\Psi$. The last inequality then yields the first relation in (3).

To obtain the remaining inequality in (3), it suffices to write any $\Psi \in \operatorname{SO}(m)$ as $\Phi\left(\theta_{1}, \ldots, \theta_{p}\right)$, which acts as the identity on $\left[\Pi_{1} \oplus \ldots \oplus \Pi_{p}\right]^{\perp}$, and as a rotation by the angle $\theta_{l} \in[-\pi, \pi]$ on each $\Pi_{l}$, for some fixed set of mutually orthogonal planes $\Pi_{l}$ in $\mathbf{R}^{m}, l=1, \ldots, p$. The length of the curve $[0,1] \ni t \mapsto \Phi\left(t \theta_{1}, \ldots, t \theta_{p}\right)$, joining $I$ to $\Psi$, is then $\left[\theta_{1}^{2}+\cdots+\theta_{p}^{2}\right]^{1 / 2} \leq \sqrt{k / 2} \pi$.

Remark 4 . The upper bounds in (3) reflect the following easily verified facts, which we do not use: the metric on $\mathrm{SO}(m)$ with the distance function dist is bi-invariant, the dist-diameter of $\mathrm{SO}(m)$ equals $\sqrt{k / 2} \pi$, for $k$ as in (3), and the curve joining $I$ to $\Psi$ in $\mathrm{SO}(m)$, defined above, is a minimizing geodesic.

\section{Proof of Theorem 1}

Given a compact orientable Riemannian manifold $(M, g)$ with a $C^{\infty}$ vector field $w$ satisfying (11), let us choose a constant $c>0$ with $\mathcal{L}_{w} g+\operatorname{Ric} \geq c g$. We then have the following upper bound on the length spectrum:

$$
L \leq \sqrt{k / c} \pi \text { for the largest even integer } k \leq \operatorname{dim} M-1,
$$

$L$ being the length of any smoothly closed constant-speed geodesic $[a, b] \ni t \mapsto x(t)$ in $(M, g)$ which represents a local minimum of the length functional in its free homotopy class. Namely, (3) with $\mathcal{L}_{w} g+$ Ric $\geq c g$ gives

$$
c L^{2}=(b-a) c \int_{a}^{b} g(\dot{x}, \dot{x}) d t \leq(b-a) \int_{a}^{b} \operatorname{Ric}(\dot{x}, \dot{x}) d t \leq k \pi^{2},
$$

and (4) follows. (The Lie-derivative term in (2) does not contribute to the integral, as $\left(\mathcal{L}_{w} g\right)(\dot{x}, \dot{x})=2 d[g(w, \dot{x})] / d t$. $)$

The uniform bound (4) implies in turn that there are only finitely many free homotopy classes of closed curves in $M$. In fact, an infinite sequence of smoothly closed geodesics $\gamma_{j}$ with uniformly bounded lengths $L_{j}$ cannot represent an infinite number of distinct free homotopy classes, as one sees choosing a point $x_{j}$ on each $\gamma_{j}$ with a unit vector $u_{j}$ tangent to $\gamma_{j}$ at $x_{j}$, and then selecting a convergent subsequence of the sequence $\left(x_{j}, u_{j}, L_{j}\right)$.

Since the free homotopy classes are in a bijective correspondence with the conjugacy classes in the fundamental group of $M$, this completes the proof. 


\section{REMARKS}

In this section we elaborate on some comments made in the Introduction.

Ricci solitons on a compact manifold $M$ are precisely the fixed points of the Ricci flow $d g / d t=-2$ Ric projected, from the space of metrics, onto its quotient under diffeomorphisms and scalings [7, [12. For shrinking solitons those scalings cause the metric to shrink to zero in finite time. Theorem 1 of 8$]$ states that, under the Ricci flow with any initial metric $g$ on a compact manifold $M$, the lengths of curves representing a fixed element of infinite order in $H_{1}(M, \mathbf{Z})$ remain bounded away from zero. As a consequence, if $(M, g)$ is a compact shrinking Ricci soliton, $H_{1}(M, \mathbf{Z})$ has no element of infinite order, and so it is finite.

Finally, compact shrinking Ricci solitons $(M, g)$ in which $g$ is a Kähler metric are known to be simply connected. In fact, as shown by Perelman [12, Remark 3.2], in a compact Ricci soliton $(M, g)$ a vector field $w$ with (2) may always be replaced by a gradient. If $g$ is Kähler (so that Ric is Hermitian), $w$ must therefore be holomorphic. The real cohomology classes of the Ricci and Kähler forms of $g$ then are related by $[\rho]=c[\Omega]$, as (22) gives $\rho=c \Omega-d \xi$ for the 1 -form $\xi=\imath_{w} \Omega$. Hence $c_{1}(M)>0$, and so $\pi_{1} M=\{0\}$ due to a result of Kobayashi [1, Theorem 11.26].

\section{REFERENCES}

[1] A. L. Besse, Einstein Manifolds, Ergebnisse (3) 10, Springer-Verlag, Berlin, 1987. MR0867684 (88f:53087)

[2] H.-D. Cao, Existence of gradient Kähler-Ricci solitons, Elliptic and parabolic methods in geometry (Minneapolis, MN, 1994), 1-16, A. K. Peters, Wellesley, MA, 1996. MR 1417944 (98a:53058)

[3] A. Derdzinski, Compact Ricci solitons, in preparation.

[4] M. Feldman, T. Ilmanen and D. Knopf, Rotationally symmetric shrinking and expanding gradient Kähler-Ricci solitons, J. Differential Geom. 65 (2003), 169-209. MR2058261 (2005e:53102)

[5] M. Fernández-López and E. García-Río, A remark on compact Ricci solitons, preprint.

[6] D. H. Friedan, Nonlinear models in $2+\varepsilon$ dimensions, Ann. Physics 163 (1985), 318-419. MR0811072 (87f:81130)

[7] R. S. Hamilton, The Ricci flow on surfaces, in: Mathematics and general relativity (Santa Cruz, CA, 1986), 237-262, Contemp. Math., Vol. 71, Amer. Math. Soc., Providence, RI, 1988. MR0954419 (89i:53029)

[8] T. Ilmanen and D. Knopf, A lower bound for the diameter of solutions to the Ricci flow with nonzero $H^{1}\left(M^{n} ; \mathbf{R}\right)$, Math. Res. Lett. 10 (2003), 161-168. MR.1981893 (2004a:53085)

[9] T. Ivey, Ricci solitons on compact three-manifolds, Differential Geom. Appl. 3 (1993), 301307. MR1249376 (94j:53048)

[10] N. Koiso, On rotationally symmetric Hamilton's equation for Kähler-Einstein metrics, Recent topics in differential and analytic geometry, 327-337, Adv. Stud. Pure Math., 18-I, Academic Press, Boston, MA, 1990. MR/1145263 (93d:53057)

[11] J. Makowsky, On some conjectures connected with complete sentences, Fund. Math. 81 (1974), 193-202. MR0366647(51:2894)

[12] G. Perelman, The entropy formula for the Ricci flow and its geometric applications, preprint, http://arXiv.org/abs/math.DG/0211159

[13] X. J. Wang and X. Zhu, Kähler-Ricci solitons on toric manifolds with positive first Chern class, Adv. in Math. 188 (2004), 87-103. MR2084775 (2005d:53074)

Department of Mathematics, Ohio State University, Columbus, Ohio 43210

E-mail address: andrzej@math.ohio-state.edu 